\title{
Lung Adenocarcinoma with Gingival Metastasis
}

\author{
Imen Gargouri ${ }^{1}$, Wafa Hergli Benzarti ${ }^{1}$, Sana Aissa $^{1}$, Samia Kanoun-Belajouza ${ }^{2}$, Abdelaziz Hayouni ${ }^{1}$, \\ Abdelhamid Garrouche ${ }^{1}$, Ahmed Abdelghani ${ }^{1}$, Mohamed Benzarti ${ }^{1}$ \\ ${ }^{1}$ Pneumology Department, Farhat Hached University Hospital, Sousse, Tunisia \\ ${ }^{2}$ Radiotherapy Department, Farhat Hached University Hospital, Sousse, Tunisia
}

Received: 05/02/2017

Accepted: 05/03/2018

Published: 10/04/2018

How to cite this article: Gargouri I, Benzarti WH, Aissa S, Kanoun-Belajouza S, Hayouni A, Garrouche A, Abdelghani A, Benzarti M. Lung adenocarcinoma with gigival metastasis. EJCRIM 2018;5: doi:10.12890/2018_000861.

Conflicts of Interests: The Authors declare that there are no competing interests.

This article is licensed under a Commons Attribution Non-Commercial 4.0 License

\section{ABSTRACT}

Gingival metastasis of lung cancer is uncommon. We report the case of an 82-year-old male smoker admitted to the pulmonology department with right pleural effusion. A chest computed tomography (CT) scan showed an invasive right hilar tumour, adherent to the superior vena cava, pulmonary artery, main right bronchus, mediastinal pleura and pericardium with lymphangitic carcinomatosis of the right lung. Pleural biopsy revealed pleural metastasis of pulmonary adenocarcinoma, its primary lung origin being confirmed by immunohistochemistry. One month later, the patient developed an ulcerated polypoid gingival mass. Biopsy of this lesion showed a poorly differentiated carcinoma compatible with metastasis from the lung adenocarcinoma. The patient underwent irradiation of the gingival mass at a dose of $30 \mathrm{Gray}$, but his condition worsened rapidly and he was not fit for chemotherapy. He received palliative treatment and died 2 months after diagnosis of his metastatic lung cancer.

\section{LEARNING POINTS}

- Lung cancers mostly metastasize to the bones, liver, lymph nodes, brain, lung and adrenal glands, with adenocarcinoma being the most common histological type.

- Distant metastasis to the oral region is very uncommon but can be the first manifestation of a primary tumour.

- Oral metastasis can be mistaken for a benign lesion, so a biopsy should be taken for further analysis.

\section{KEYWORDS}

Lung adenocarcinoma, gingival metastasis

\section{INTRODUCTION}

Metastatic cancers to the oral region from distant sites are very unusual, and are more often mandibular metastases than the rare gingival metastases $^{[1]}$. The clinical diagnosis of a gingival metastasis is generally difficult, especially in the absence of an obvious primary tumour, and must be confirmed by histological examination. Because of their similarity to benign lesions, the prevalence of oral metastases could be underestimated. We report a case of gingival metastasis of lung adenocarcinoma. 


\section{CASE REPORT}

An 82-year-old male smoker was admitted to the pulmonology department because of a 1-month history of right pleuritic chest pain, exertional dyspnoea and weight loss. On examination, patient was stable with a performance status (PS) of 1 and had right-sided pleural effusion.

A chest computed tomography (CT) scan showed an expansive right hilar tumour adherent to the superior vena cava, pulmonary artery, main bronchus $1 \mathrm{~cm}$ above the carena, mediastinal pleura and pericardium with signs of lymphangitic carcinomatosis of the right lung (Fig. 1). Pleural fluid analysis following aspiration revealed an exudative lymphocytic pleural effusion. Pleural biopsy revealed pleural metastasis of pulmonary adenocarcinoma; the primary pulmonary origin was confirmed by immunohistochemistry.

The patient developed an indurated and ulcerated polypoid mass along his maxillary gingiva 1 month after detection of the primary tumour (Fig. 2). An excisional biopsy of this mass was carried out and the histopathology analysis demonstrated a poorly differentiated carcinoma compatible with metastasis from the bronchial adenocarcinoma.

The patient received irradiation of the gingival lesion at a dose of 30 Gray but his condition worsened rapidly. He was unable to receive chemotherapy due to his low PS and died 2 months later.

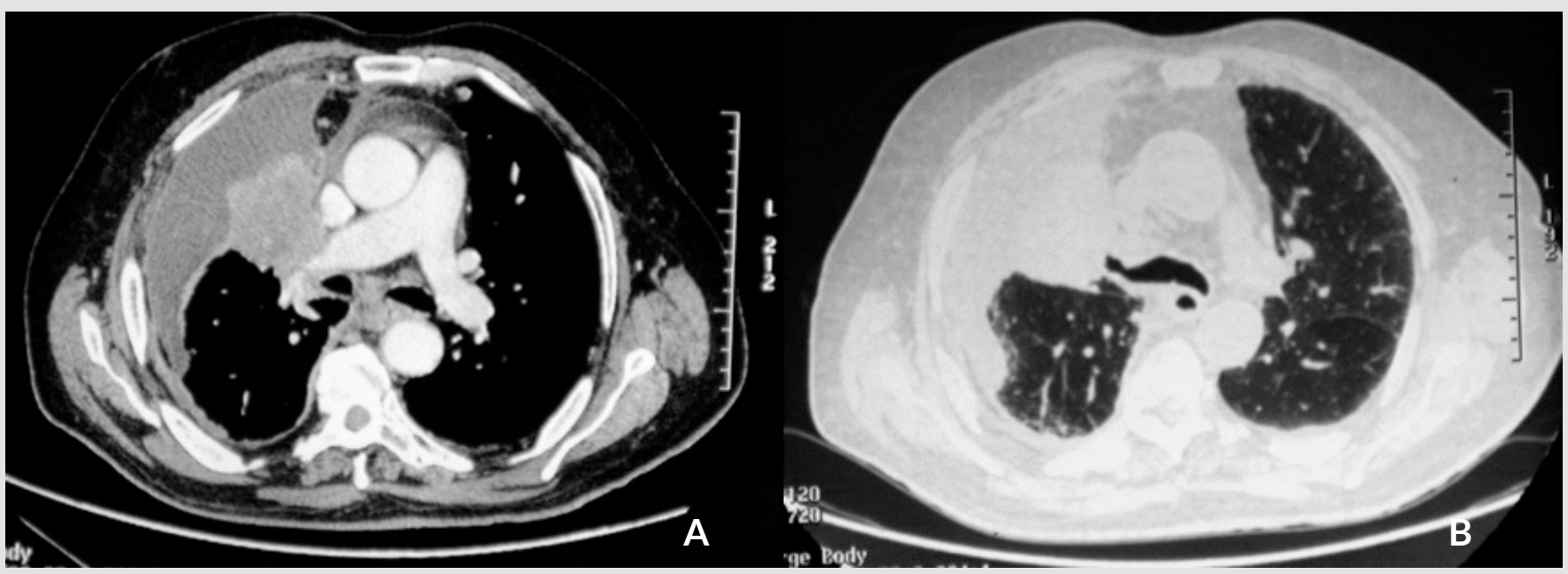

Figure $1(a, b)$. CT scan showing a right hilar tumour adherent to the superior vena cava, pulmonary artery, bronchus $1 \mathrm{~cm}$ above the carena, mediastinal pleura and pericardium with signs of carcinomatous lymphangitis of the right lung

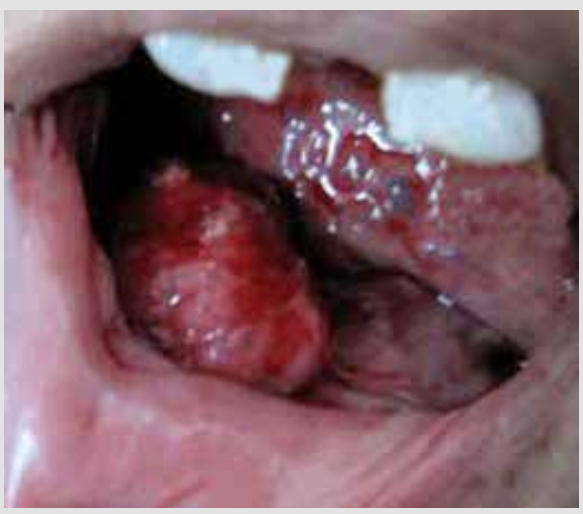

Figure 2. Ulcerated polypoid gingival mass in a patient recently diagnosed with lung adenocarcinoma

\section{DISCUSSION}

Metastatic cancers to the oral region are very unusual: Meyer and Shklar reported that only 25 of over 2,400 malignant oral cavity tumours were metastatic ${ }^{[2]}$. Metastases to soft tissue are much less common than to bone. 
The most common sites of soft tissue metastases to the oral cavity are the gingiva (54\%) and alveolar mucosa, followed by the tongue ${ }^{[3]}$. The problem with gingival metastasis is the clinical distinction between a benign and a malignant lesion. Another problem is the difficulty in distinguishing between a primary tumour and a metastasis. The final diagnosis is based on histopathology and must be confirmed by immunohistochemistry.

In $30 \%$ of cases, oral metastasis is the first manifestation of the cancer ${ }^{[4]}$ and usually indicates an advanced, multiple-metastatic cancer. In a review of 157 metastases to the oral mucosa in men, the primary tumour was in the lung in $36 \%$ of cases, the kidney in $16 \%$ and the skin in $15 \%$. In female patients, oral metastatic tumours most commonly originate from breast tumours (24\%), followed by gynaecological (17\%), lung (12\%), bone (10.3\%) and renal tumours (10.3\%) ${ }^{[4]}$.

The pathogenesis of oral metastatic tumours is not yet fully understood. According to the literature, haematogenous spread is most likely. Local inflammation promotes the development of metastases as the spread of metastatic cells is facilitated by the greater permeability of vessels and the presence of adhesive molecules ${ }^{[5]}$. Another possible mechanism is grafting of tumour cells in the sputum to gingival mucosa weakened by microtrauma ${ }^{[6]}$.

The clinical appearance is often misleading and unspecific and reminiscent of a benign disease such as a periodontal abscess, or of a tumour pathology such as haemangioma or lymphoma ${ }^{[6]}$.

Gingival metastases are generally described as polypoid or exophytic, are highly vascularized and haemorrhage is very often found ${ }^{[5]}$. Clinical suspicion must be confirmed by immunohistochemistry. More than $90 \%$ of these tumours have a CK $7+$ and CK $20-$ phenotype, and show the TTF-1 expressed by two-thirds of primary adenocarcinomas of the lung ${ }^{[7]}$.

The median survival time of lung cancer patients with gingival metastasis is only 4 months as the prognosis is very poor ${ }^{[8]}$. Gingival metastasis is treated with systemic chemotherapy or radiotherapy, which is not particularly effective. However, surgical excision under local anaesthesia provides rapid relief of symptoms and improves dental function ${ }^{[9]}$.

\section{CONCLUSION}

Gingival metastases often have a benign appearance and so can be misdiagnosed, compromising treatment. Excisional biopsy is necessary for diagnosis and has the potential to simultaneously restore oral function and improve nutritional status.

\section{REFERENCES}

1. Jaguar GC, Prado JD, Soares F, Alves FA, Toleda Osório CA. Gingival metastasis from non-small cell undifferentiated carcinoma of the lung mimicking a pyogenic granuloma. Oral Oncol Extra 2006;42:36-39.

2. Meyer I, Shklar G. Malignant tumors metastatic to mouth and jaws. Oral Surg Oral Med Oral Pathol 1965;20:350-362.

3. Hirshberg A, Leibovich P, Buchner A. Metastases to the oral mucosa: analysis of 157 cases. J Oral Pathol Med 1993;22:385-390.

4. Hirshberg A, Leibovich P, Buchner A. Metastatic tumors to the jawbones: analysis of 390 cases. J Oral Pathol Med 1994;23:337-341.

5. Amro L, Maliki L, Belabidia B, Yazidi AA. Gingival metastasis revealing lung adenocarcinoma. Adv Lung Cancer 2014;3:35-37.

6. Elias D. Basic concepts and future prospects of oncologic surgery. Ann Chir 1997;51:21-31.

7. Watanabe E, Touge H, Tokuyasu H, Kawasaki Y. Gingival metastasis of adenocarcinoma from the lung. Respir Med CME 2008;1:103-106.

8. Tanaka M, Hiraki A, Ueoka H, Bessho A, Kiura K, Takigawa N, et al. Gingival metastasis in lung cancer. Oncol Rep 2002;9:571-574.

9. Huang C-J, Chang Y-L, Yang M-C, Hsueh C, Yu C-T. Lung cancer metastatic to the maxillary gingiva-a case report and literature review. Oral Oncol Extra 2005;41:118-120. 